\title{
Analytical gear mesh model using 3D gear geometry
}

\author{
Z. Neusser*, T. Vampola**, M. Valášek*** \\ ${ }^{*}$ Czech Technical University in Prague, Faculty of Mechanical Engineering, Technická 4, Prague 6, 166 07, Czech \\ Republic, E-mail: zdenek.neusser@fs.cvut.cz \\ **Czech Technical University in Prague, Faculty of Mechanical Engineering, Technická 4, Prague 6, 166 07, Czech \\ Republic, E-mail: tomas.vampola@fs.cvut.cz \\ ***Czech Technical University in Prague, Faculty of Mechanical Engineering, Technická 4, Prague 6, 166 07, Czech \\ Republic, E-mail: michael.valasek@fs.cvut.cz.
}

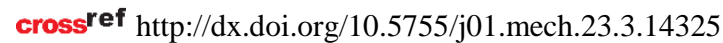

\section{Introduction}

The necessity to have a gear mesh model covering all aspects of gear teeth shape imperfections, gear wheel deformations and shaft misalignments is obvious when estimating vibrations especially at higher frequencies. It is also useful for the prediction of the transmission error of a gearbox.

Quasi-static simulation is presented in [1] for a gear pair. The tooth deformation is calculated using finite element method and using approximate interpolation functions it can react to any external load and resulting contact pressure is evaluated based on the Hertz' theory. The method needs FE analysis of tooth with wheel and tooth flank deformation approximation for teeth pair. The paper presents only steady state case. These are significant drawbacks of the presented method, even if it can be fast and accurate enough. In the work [2] is presented 2D approach with necessity of having FE model for teeth compliancy evaluation. The contact zones are analytically calculated reflecting real teeth creation process. The method is presented only using quasi-static simulation and requires finite element model evaluation. Slightly different approach is used in [3]. It presents lumped-parameter gear mesh model with stiffness distribution along the tooth, which is covered in the mesh stiffness matrix. The static simulation of developed model is compared with the FE model. The stiffness matrix is obtained from computational contact algorithm, it needs pre-calculation and only static simulation is shown. The application of the simple springmass model of gear meshing is demonstrated in [4]. Precalculated 3D contact stiffness is the basis for the gear joint element proposed in [5]. The gear joint element covers many details of gear meshing process and stores this information in pre-calculated lookup table. The FE model of gear pair creation and simulation is presented in [6]. The contact surfaces have normal interface springs between all penetrating nodes and high amount of contact details could be covered. Model is automatically and parametrically created, but the simulation time is questionable. Tooth design by tool shaping using simulation is shown in [7] with stress analysis reflecting tool shape modifications. The work shows FE model stress calculation comparison with analytical solution. The pair of gear teeth is represented by multiple springs in [8], gear meshing details are neglected. Gear mesh simulated as a spring with backlash is used in [9] for a helical planetary gear set. The model consists of many gear connections and is computationally efficient, but the gear meshing process events (multiple teeth in contact, teeth modifications and corrections etc.) are not covered. The FE model method is reduced and transferred into the frequency domain in [10] with contact treated semi-analytically. The details in teeth contact are not evaluated. The gear mesh model can be simplified as a transmission error scalar equation and for quasi-static evaluation is presented in [11].

There are some model variants, but in general the gear mesh models can be sorted into three groups. The first one is the spring-damper representation, the second is the FE model of gear modeling with contact between teeth and the third is the analytical formula of contact. There are some modifications but generally it could be stated, that from the aspect of computation time, the simple spring model is the fastest one, the FE model is the slowest but more precise one and the analytical solution is somewhere between them.

This paper shows the simple dynamic gear mesh model extended with an advanced 3D tooth contact kinematic model. Such a hybrid model gives much more information about the tooth contact behaviour, with a computational speed higher than that in the related FE based model. Also measured teeth flank modifications are applied. The contact kinematic model uses an ideal tooth shape with modifications for introducing multiple teeth contact, teeth engagement, and generally teeth whine effects.

The gear contact is in fact an example of the flexible joint as a gear wheel being deformed is a compliant body fulfilling the function of movable connection of other bodies. The gearbox with deformed gear wheels is a compliant mechanism. This paper presents another way of description of compliant mechanism.

\section{Model overview}

The model of a gearbox is in Fig. 1. The parameters of the mating gears are: normal module $m_{n}$, pinion and wheel number of teeth $z_{p}$ and $z_{w}$, normal pressure angle $\alpha_{n}$, pitch helical angle $\beta_{p}$, center distance $d$, pinion and wheel moments of inertia $I_{p}$ and $I_{w}$, pinion and wheel loads $M_{p}$ and $M_{w}$, teeth contact stiffness $K$ and damping $D$. The interaction force between the teeth is represented by the force $F_{c}$. This interaction could be denoted as the gear mesh force, which is calculated from the teeth penetration and the teeth contact stiffness.

The way how to calculate teeth penetration is the main contribution of this paper. The contact force $F_{c}$ is evaluated applying the spring-damper model in Fig. 1 using Eq. (3), where $\Delta p$ and $\Delta p$ are the penetration and penetration 


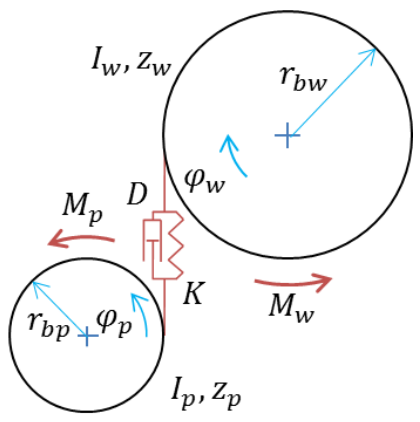

Fig. 1 Gearbox model

time derivative respectively. The penetration $\Delta p$ is analyzed out on backlash presence. Eqs. (1) and (2) are equations of gear motion. Angles $\varphi_{p}$ and $\varphi_{w}$ are measured from the gear centers connection line, $I_{p}$ and $I_{w}$ are inertia moments of the pinion and wheel respectively and $M_{p}$ and $M_{w}$ are the gear external loads:

$$
\begin{aligned}
& I_{P} \ddot{\varphi}_{P}=M_{P}-F_{C} r_{P}, \\
& I_{W} \ddot{\varphi}_{W}=-M_{W}+F_{C} r_{W}, \\
& F_{C}=K \Delta p+D \Delta \dot{p} .
\end{aligned}
$$

The calculation of the tangential values of module $m$, pressure angle $\alpha$, helical angle $\beta$ on the base circle, pinion $r_{b p}$ and wheel $r_{b w}$ base radii are evaluated in equations (4), (5), (6), (7) and (8) respectively:

$$
\begin{aligned}
& m=\frac{m_{n}}{\cos \left(\beta_{P}\right)}, \\
& \alpha=\operatorname{atan} \frac{\tan \left(\alpha_{n}\right)}{\cos \left(\beta_{P}\right)}, \\
& \beta=\operatorname{atan} \frac{\tan \left(\beta_{P}\right)}{\cos (\alpha)}, \\
& r_{b p}=\frac{m z_{p} \cos (\alpha)}{2}, \\
& r_{b w}=\frac{m z_{w} \cos (\alpha)}{2} .
\end{aligned}
$$

\section{Penetration model based on simplified gear geometry}

The first approach which calculates gear pair penetration uses the gear angular position and base radius of the pinion and wheel, Fig. 2. The penetration is evaluated as the difference between the arcs of rotation of both gears in Eq. (9), characterized by gear angles $\varphi_{p}$ and $\varphi_{w}$ and base gear radii. Base radii $r_{b p}$ (pinion) and $r_{b w}$ (wheel) are calculated using Eqs. (7) and (8) respectively. The backlash is involved modifying Eq. (9) with the penetration magnitude comparison to the half size of teeth gap. According to the comparison result, the penetration value is the half size of teeth gap lowered or zero, see Eq. (10). The penetration derivative $\Delta p$ is the time derivative of $\Delta p$. The angles $\varphi_{p}$ (pinion) and $\varphi_{w}$ (wheel) are obtained by solving dynamic Eqs. (1) and (2):

$$
\Delta p=r_{b p} \varphi_{p}-r_{b w} \varphi_{w},
$$

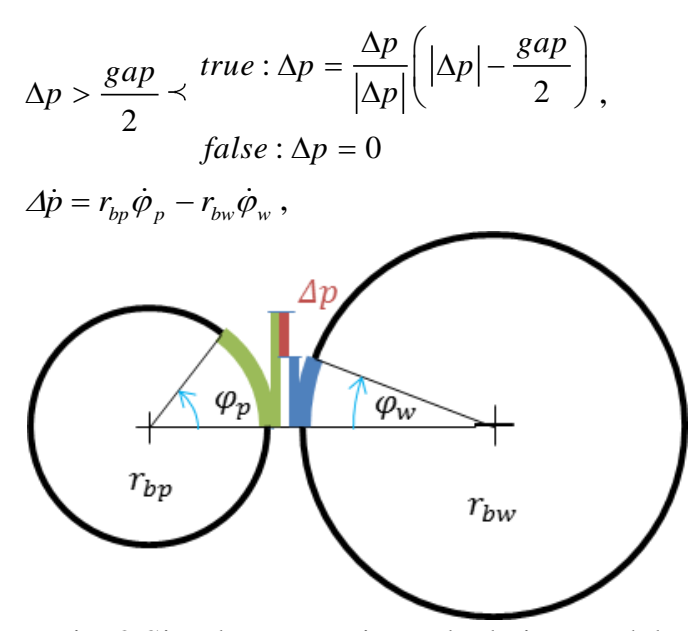

Fig. 2 Simple penetration calculation model

\section{Penetration model based on 3D gear geometry}

The second approach to penetration calculation uses the full 3D geometry of gears with teeth. Penetration is calculated along the intersection lines between all mating teeth, and such theoretical intersection lines between teeth are placed in the plane of action, which is a tangent to the base cylinders of both mating gears (Fig. 3).

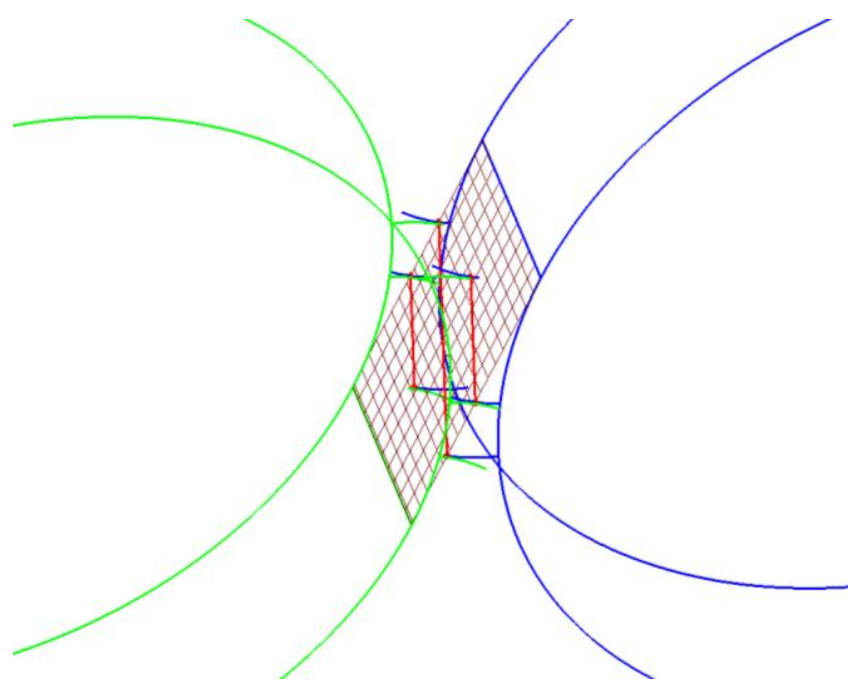

Fig. 3 Intersection lines of mating teeth

\subsection{Gear parameterization}

Primarily it is necessary to describe the theoretical gear flank shape. General flank parameterization is made for the contact evaluation. The angle of the tooth flank from the gear centers connection line consists of the gear rotation $\varphi$ and the angle $\varphi_{h}$ due to the inclination of the helical gear shape (Fig. 4). The angle $\varphi_{h}$ using Eq. (12) could be derived from the axial contact width of the gear $b$, helical angle $\beta$ and base radius $r_{b}$. The axial width of the gear is parameterized by the parameter $t_{l}$, which ranges from one side with a zero value to the opposite one with value one (Fig. 4).

$$
\varphi_{h}=\frac{b t_{1} \tan (\beta)}{r_{b}} .
$$


The base radius is evaluated using Eqs. (7) and (8). The theoretical shape of the tooth flank could also be parameterized. The parameterization is based on the angle $\chi$ from Fig. 5. The flank starts at the base circle $(\chi=0)$ or at some general point $\left(\chi=\chi_{\text {start }}\right)$, where the parameter $t_{2}$ equals zero. The final point of the flank is shown in Fig. 5 anchored by angle $\chi_{\text {end }}$. This point is situated on the head circle of the gear and $t_{2}$ equals one. The flank parameterization is expressed by the angle $\chi$ - see Eq. (13):

$$
\chi=\chi_{\text {start }}+t_{2}\left(\chi_{\text {end }}-\chi_{\text {start }}\right) \text {. }
$$

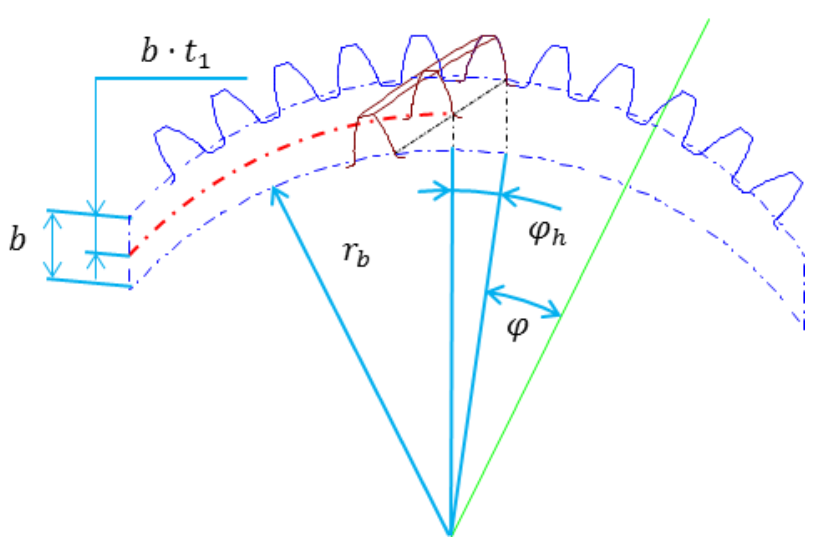

Fig. 4 Gear axial parameters

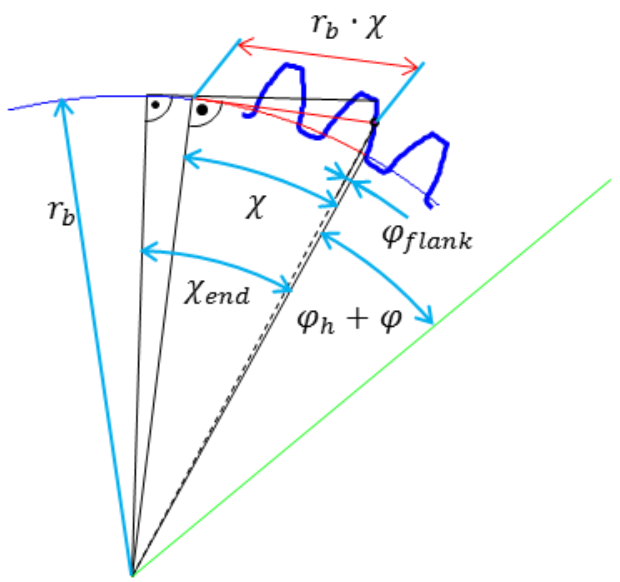

Fig. 5 Gear radial parameters

In Eq. (13) the angle $\chi_{\text {start }}$ is obtained using the radius of the active flank beginning $r_{\text {start }}$, see Eq. (14) and $\chi_{\text {end }}$ is calculated from the head circle radius $r_{a}$ according to Eq. (15):

$$
\begin{aligned}
& \chi_{\text {start }}=\sqrt{\left(\frac{r_{\text {start }}}{r_{b}}\right)^{2}-1}, \\
& \chi_{\text {end }}=\sqrt{\left(\frac{r_{a}}{r_{b}}\right)^{2}-1} .
\end{aligned}
$$

Finally, the flank point position in the plane cut is defined by its angle $\varphi_{\text {flank }}$ (Eq. (16)) and the distance from the gear center $R_{\text {flank }}$, see Eq. (17):

$$
\begin{aligned}
& \varphi_{\text {flank }}=\chi-\operatorname{atan}(\chi), \\
& R_{\text {flank }}=r_{b} \sqrt{1+\chi^{2}} .
\end{aligned}
$$

Flank parameterization in variables $t_{1}$ and $t_{2}$ is shown in Fig. 6.

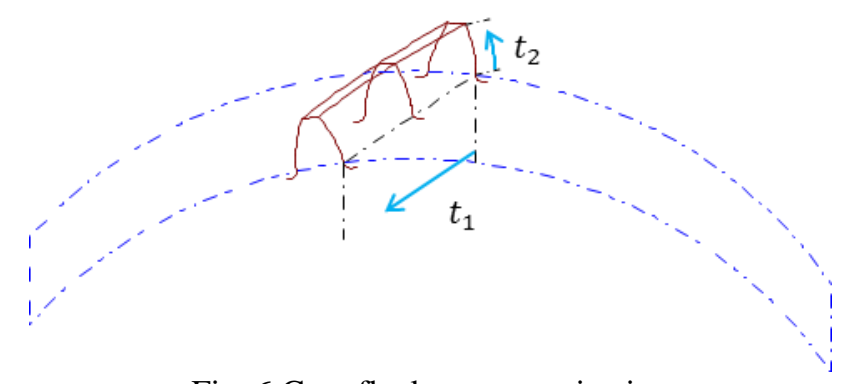

Fig. 6 Gear flank parameterization

\subsection{Contact position}

The contact of two theoretical rigid gears is situated on the plane of action (Fig. 3). This plane is deduced from the involute tooth shape. The involute designs the flank in such a way that the normal vectors of all flank points are tangent to the base circle (base cylinder). This is why the theoretical contact points must be located tangentially to both base cylinders of the mating gears.

Fig. 7 shows two gears in the plane. All theoretical contact points are located on the line of action. Location of the contact points in space is on the plane of action, which is a tangent to both base cylinders, as it is shown in Fig. 3.

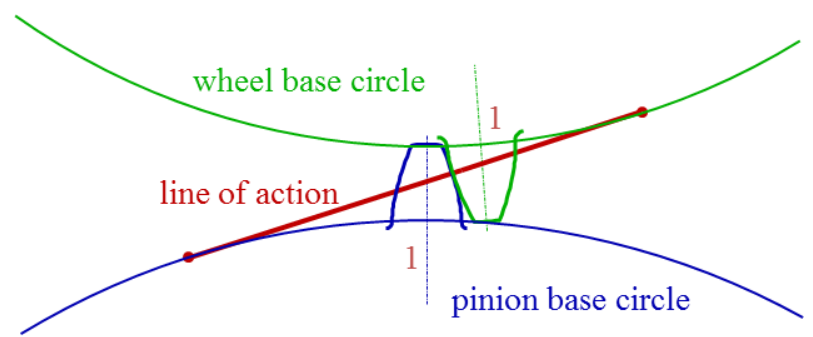

Fig. 7 Contact point location on the plane cut - line of action

\subsection{Contact lines}

Because all theoretical contact points are situated only on the plane (line) of action, it is necessary to find the intersecting lines between the tooth flanks and the plane of action. Because the intersecting lines are straight lines, it is sufficient to find just their corner points. The corner points of the intersecting lines are obtained as intersections between plane of action and the border lines of flanks. In order to detect which flank border lines intersect with the plane of action, the corner point directions from the plane of

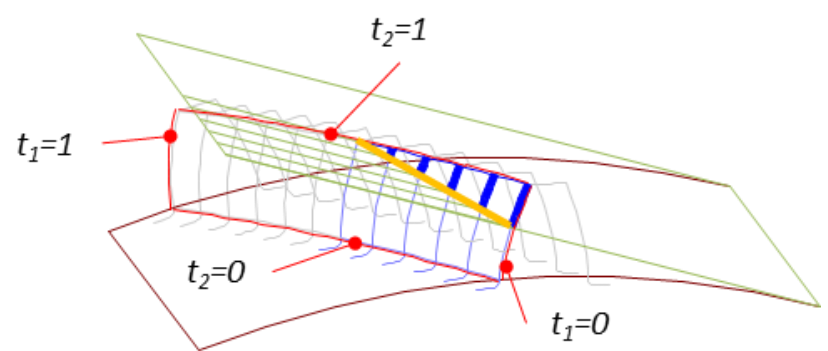

Fig. 8 Intersection between the tooth flank and the plane of action 
action are evaluated. If two adjacent corner points are in the opposite half-space split by the plane of action, the involved flank border line has an intersection with the plane of action. The situation is shown in Fig. 8, where the penetration line between the tooth flank and the plane of action is the bold line.

From Fig. 8 it is obvious that the starting point of penetration corresponds with $t_{1}=0$ and the terminal point corresponds with $t_{2}=1$, and for such values the corresponding parameterization is calculated, see equations (18) and (19):

$$
\begin{aligned}
& t_{1}=0 \ldots t_{2}=\frac{\alpha-\chi_{\text {start }}+\varphi}{\chi_{\text {end }}-\chi_{\text {start }}}+\frac{t_{1} b \tan (\beta)}{r_{b}\left(\chi_{\text {end }}-\chi_{\text {start }}\right)}, \\
& t_{2}=0 \ldots t_{1}=\frac{r_{b}\left(\alpha-\left(\chi_{\text {start }}+t_{2}\left(\chi_{\text {end }}-\chi_{\text {start }}\right)\right)-\varphi\right)}{b \tan (\beta)} .
\end{aligned}
$$

\subsection{Penetration calculation}

Knowing which flanks are in the neighbourhood (based on the number of teeth (Fig. 7), the pinion and gear contact line intersection gives final contact lines between the gears. The penetration is calculated from the distance between the penetration lines of corresponding teeth. The penetration of $i^{\text {th }}$ mating teeth pair $\Delta p_{i}$ is derived in Eq. (20) according to Fig. 9 in the face cut, where $\rho_{p}$ and $\rho_{w}$ are the distances between the tooth flank contact points of the pinion and wheel respectively and the beginning of the line (plane) of action on the pinion or wheel base circle respectively and dist is the length of the line of action calculated from the center distance $d$ and base radii $r_{b p}$ and $r_{b w}$. The gear parameters $\chi_{\text {start }}, \chi_{\text {end }}$ and $t_{2}$ have a subscript $p$ for the pinion gear flank and $w$ for the wheel gear flank and the parameter $t_{2}$ is evaluated for the contact point in Eqs. (18) and (19):

$$
\begin{aligned}
& \Delta p_{i}=\rho_{p}+\rho_{w}-\text { dist }, \\
& \rho_{p}=\left(\chi_{\text {start }_{p}}+t_{2_{p}}\left(\chi_{\text {end }_{p}}-\chi_{\text {start }_{p}}\right)\right) r_{b p}, \\
& \rho_{w}=\left(\chi_{\text {start }_{w}}+t_{2_{w}}\left(\chi_{\text {end }_{w}}-\chi_{\text {start }_{w}}\right)\right) r_{b w}, \\
& \text { dist }=\sqrt{d^{2}-\left(r_{b p}+r_{b w}\right)^{2}} .
\end{aligned}
$$

Penetration in Eq. (20) must be nonnegative (otherwise zero) which inherently covers the backlash phenomenon.

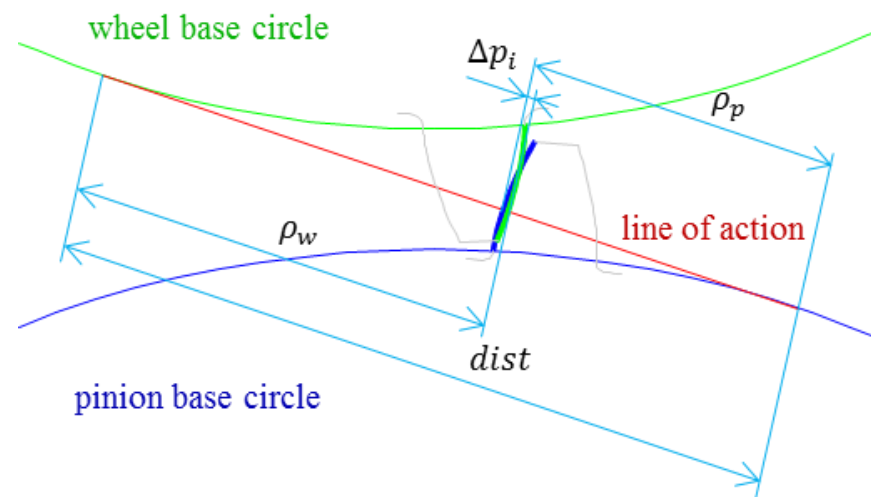

Fig. 9 Penetration calculation - 3D gear mesh model face cut
The penetration calculation presented in Eq. (20) is performed along the axial axis with parameter $t_{1}$ for all $n$ mating teeth pairs and the final penetration is in Eq. (24):

$$
\Delta p_{i}=\sum_{i=1}^{n} \int_{t_{1}} \Delta p_{i}
$$

The final penetration $\Delta p$ of two mating gears consists of each particular teeth pair penetration, because the contact force between every two teeth is evaluated using contact stiffness of two mating teeth (which is same for all pairs) with their penetration and then adding contact forces together to obtain resulting contact force.

\subsection{Tooth flank modifications}

Tooth flank modifications are used in the 3D gear mesh model. The knowledge of the real or testing teeth flank profile behaviour is necessary for applying modifications to the model. Tooth flank modifications are differences between the ideal tooth shape and the real tooth shape in the direction normal to the ideal tooth flank. The tooth flank is divided into points, where the modification value is known. The rest of the tooth flank point modification values are interpolated. Fig. 10 shows the ideal flank shape and the modified flank shape with magnified modification values in the face cut.

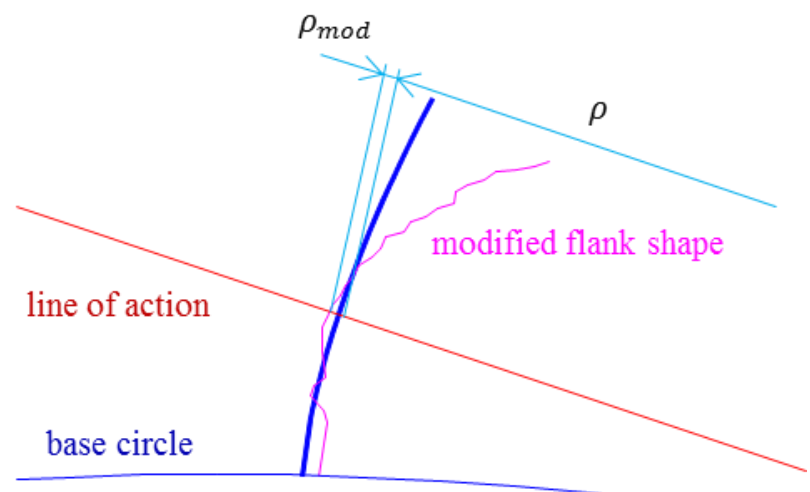

Fig. 10 Tooth flank modifications

The incorporation of tooth flank modifications into the penetration calculation in Eq. (20) is expressed in Eqs. (21) and (22). The distance $\rho$ is modified by contact point modification $\rho_{\text {mod }}$ according to Eq. (25) for both the pinion and wheel contact points. Then the total penetration is evaluated using Eq. (24):

$$
\rho=\left(\chi_{\text {start }}+t_{2}\left(\chi_{\text {end }}-\chi_{\text {start }}\right)\right) r_{b}+\rho_{\text {mod }} .
$$

\section{Results}

The workflow of calculation is shown in Fig. 11. The dynamic calculation is executed with the penetration of the teeth in contact as an input. The states are obtained and used in the penetration calculation by evaluating the contact force and by integrating dynamic equations. Penetration is obtained either using the simplified gear geometry model or by using the $3 \mathrm{D}$ gear geometry model. The contact force is then calculated from the penetration and its derivative. 
The comparison of simulation for both models is executed using the scheme in Fig. 11. The pinion gear has a prescribed acceleration and the wheel gear is loaded with white noise with a mean value $20 \mathrm{Nm}$ and amplitude $10 \mathrm{Nm}$. The Initial conditions are situated in the static equilibrium of the system with a nonzero angular velocity. The basic parameters of the gearbox are in Table 1.

Table 1

Basic gear parameters of simulated gearbox

\begin{tabular}{|l|c|c|}
\hline & Pinion gear & Wheel gear \\
\hline Number of teeth $z[-]$ & 45 & 41 \\
\hline Normal module $m_{n}[\mathrm{~mm}]$ & \multicolumn{2}{|c|}{1.65} \\
\hline $\begin{array}{l}\text { Normal pressure angle } \\
\alpha_{n}[\mathrm{deg}]\end{array}$ & \multicolumn{2}{|c|}{16} \\
\hline Helix angle $\beta_{p}[\mathrm{deg}]$ & 33 \\
\hline Center distance $d[\mathrm{~mm}]$ & 85 \\
\hline
\end{tabular}

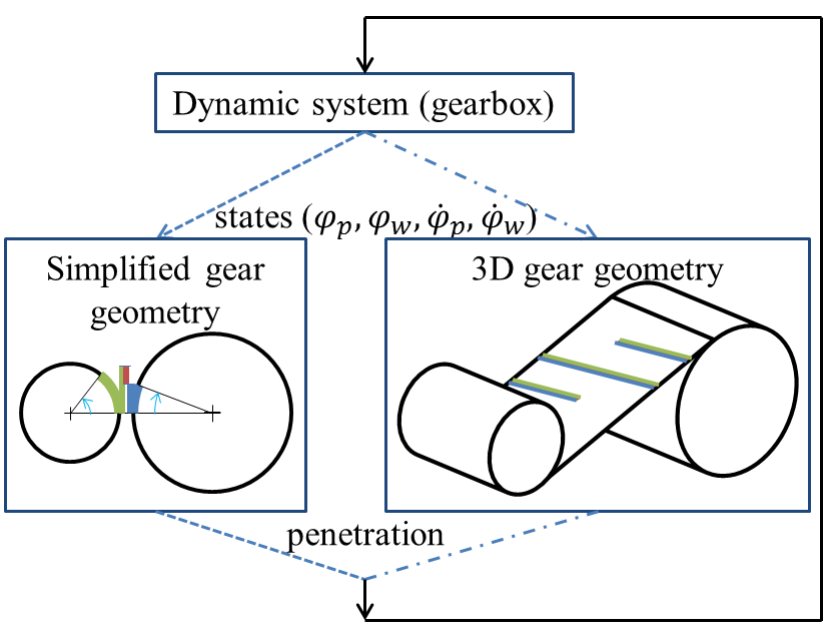

Fig. 11 Simulation workflow

The contact forces from the simplified model and 3D model are compared in graphs in the frequency domain for different pinion angular velocities. The simulation ranges from pinion angular velocity $100 \mathrm{rpm}$ to $10000 \mathrm{rpm}$.

The simulated data are displayed in Fig. 12, Fig. 13 and Fig. 14. The frequency range of interested frequencies present in the simulated signal is on the $\mathrm{x}$-axis. The pinion angular velocity in on the $y$-axis. The common logarithm of the contact force magnitude is on the $\mathrm{z}$-axis.

Some important lines are shown - the dashed line marked ' 0 ' denotes the system's eigenfrequency, line ' 1 ' denotes the $45^{\text {th }}$ order of the pinion angular velocity (which is the first tooth frequency, calculated from equation $k \frac{\omega_{p i n} z_{p}}{60}$, where $k$ is a natural number equal to 1$)$, line ' 2 ' denotes the $90^{\text {th }}$ order of the pinion angular velocity (second tooth frequency), lines ' 3 ', ' 4 ', ' 5 ', ' 6 ' represent the $135^{\text {th }}$, $180^{\text {th }}, 270^{\text {th }}, 360^{\text {th }}$ order of the pinion angular velocity $\left(3^{\text {rd }}\right.$, $4^{\text {th }}, 6^{\text {th }}$ and $8^{\text {th }}$ tooth frequency) respectively.

Evaluation of the simple gear mesh model in Fig. 12 shows only the system's eigenfrequency in the signal. Any other gear meshing behaviour is not covered.

The evaluation of the model based on 3D gear geometry includes to a great extent the system's eigenfrequency and first four tooth frequencies - see Fig. 13. The tooth frequencies are present in 3D gear geometry simulation, because the model involves teeth contact engagement and more teeth pairs in the contact simultaneously. The frequencies present in this model are easily predicable, but when a model involved in a more comprehensive multibody model is used, some new frequencies will appear (as sidebands) implied from other dominant frequencies in the multibody model, whose are related to the other elements such as bearings, clutches, another gearboxes etc. Investigated model consists only from two rotating gears and 3D gear mesh model, one gear body has kinematic constrain and the second one is loaded. That's the reason the sidebands are not present in the simulation.

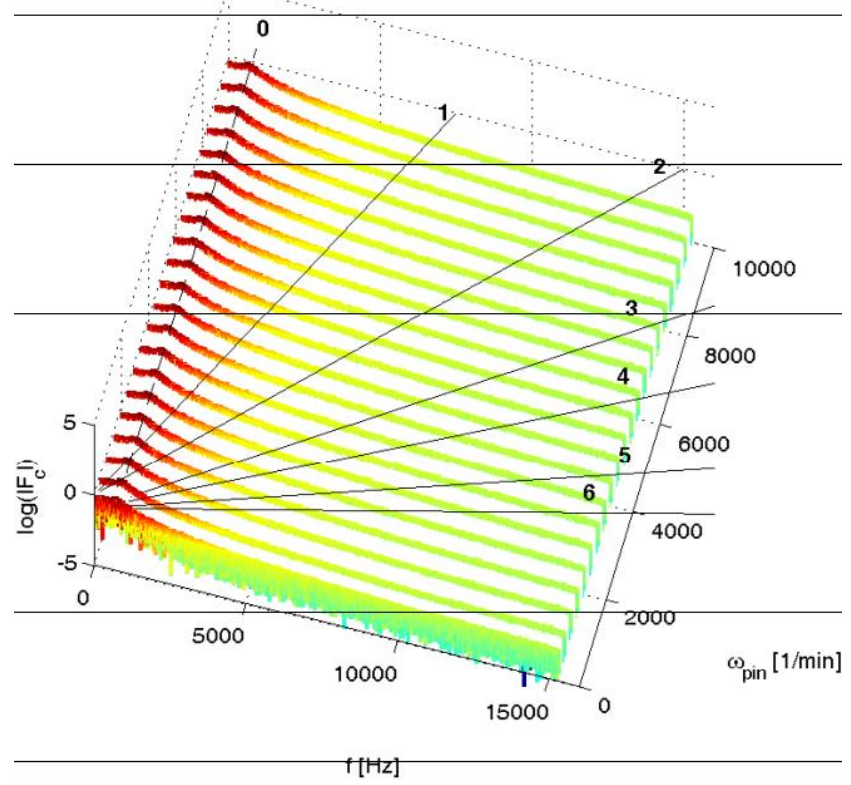

Fig. 12 Simulation using the simple gear mesh model

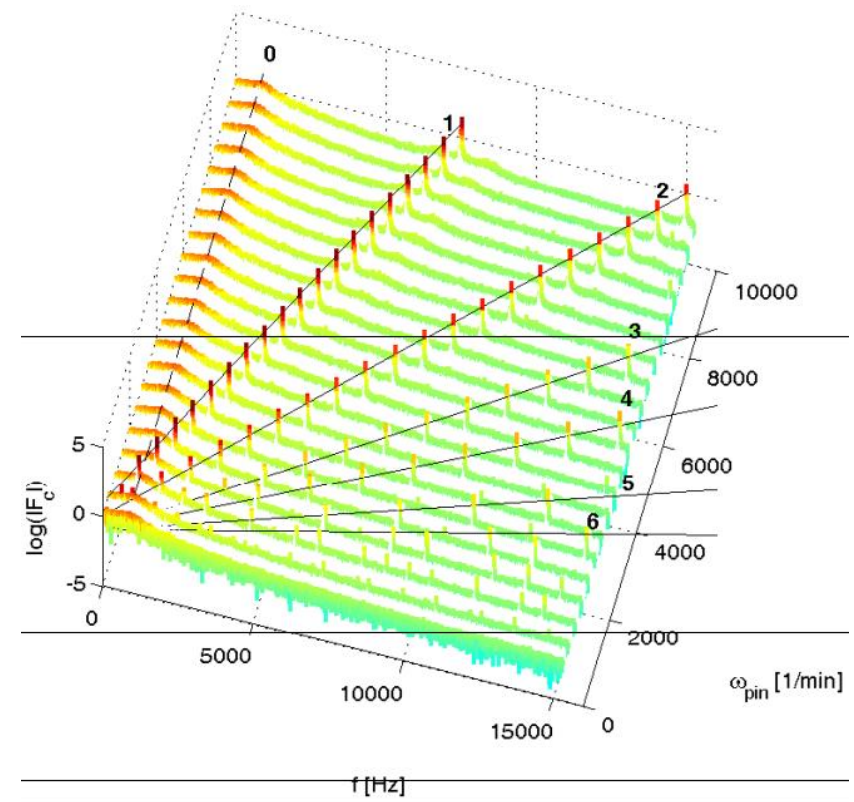

Fig. 13 Simulation with the 3D gear mesh model

The diagram in Fig. 14 shows a model based on 3D gear geometry with real profile modifications applied to the pinion and wheel gears. The model is significantly influenced by the modifications, the meshing frequencies are no longer sharply pronounced compared with the model without modifications. The model shows a much broader response up to $2000 \mathrm{~Hz}$ and there are many sidebands (lines 
marked with ' $a$ ', 'b' and 'c') which were created by the modulation of the tooth and eigenfrequency with tooth and pitch errors covered in the modifications.

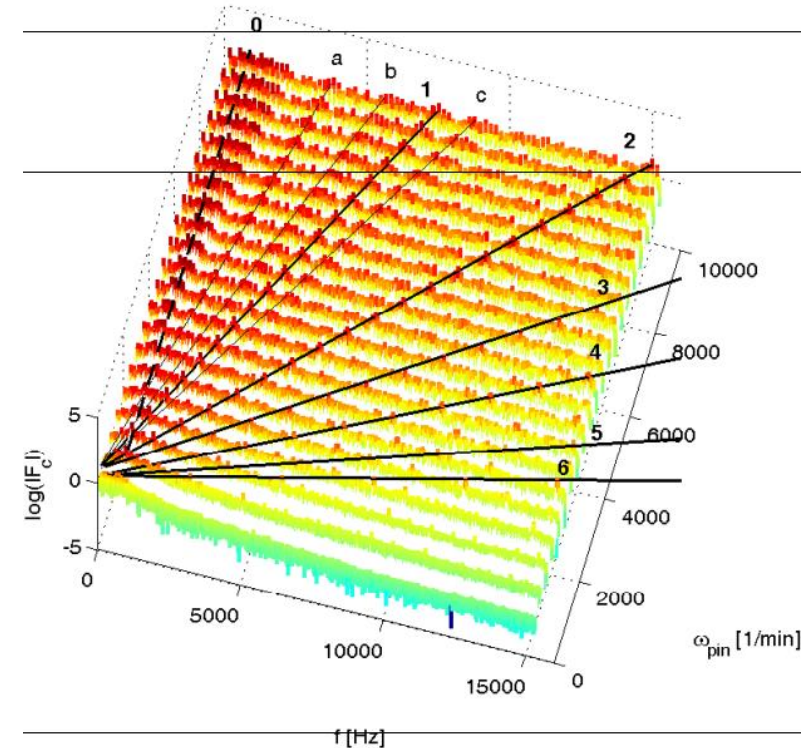

Fig. 14 Simulation with the 3D gear mesh model with tooth flank modifications

In Fig. 15 the modification values of all flanks are divided by 5 . There is an apparent drop of amplitudes along the lines marked with ' $a$ ', ' $b$ ' and 'c' and for higher frequencies. This is due to the smaller influence of tooth flank deviations from the ideal tooth profile. Fig. 16 shows the simulation with modifications applied to the tip and middle part of the tooth - the foot part of the involute flank has an ideal shape. There is even a more apparent drop of the amplitude of sideband frequencies; the amplitudes along lines ' $a$ ' and 'c' almost disappeared.

A future challenge is whether this model could be efficiently parametrized similarly as flexible bodies by the approach described in [12].

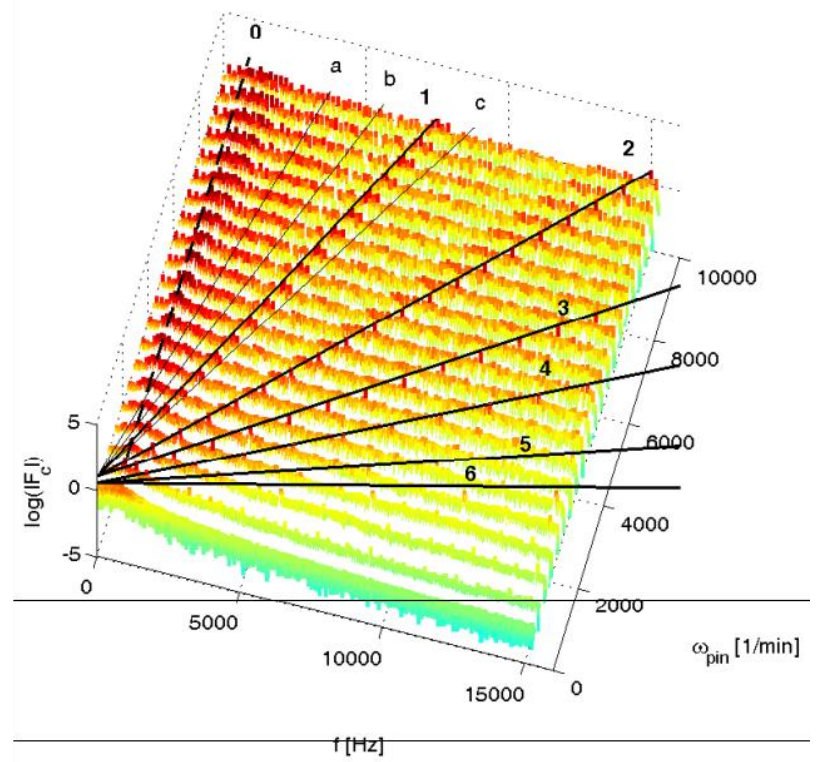

Fig. 15 Simulation with the 3D gear mesh model with reduced tooth flank modifications

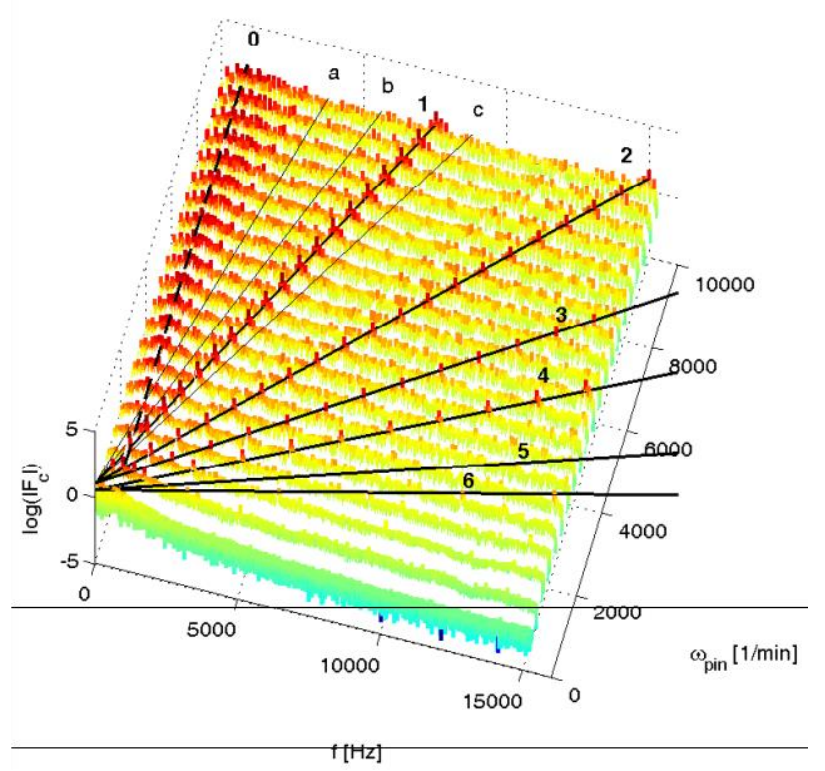

Fig. 16 Simulation with the 3D gear mesh model with partly applied tooth flank modifications

\section{Conclusions}

The 3D gear geometry model was presented and compared with the simple plane model. The testing modifications were applied to the 3D gear geometry model. The gear mesh model in 3D covers all kinematic-based events existing in the meshing process, the applied modifications show the necessity of their use for estimating broad frequency excitations. The significance of the 3D gear mesh model without modifications is in that it maintains the meshing eigenfrequencies in the contact force and propagates them further into the structure and driveline. The dynamic behaviour changes quite significantly by incorporating modifications. With such model it is possible to model gearbox noise emissions like gear rattle, hammering and gear whine and to predict transmission errors more precisely. The model shows relatively good agreement with the measurement presented in [13]. This model can be used for the design of tooth flank modifications and helps in detecting problematic frequencies in already produced gears. Reliable modifications can be suggested using this model.

\section{Acknowledgements}

This publication was supported by the project GA 101/13-39100S „Mechatronic Flexible Joint“.

\section{References}

1. Andersson, A.; Vedmar, L. 2003. A dynamic model to determine vibrations in involute helical gears, Journal of Sound and Vibration 260(2): 195-212. http://dx.doi.org/10.1016/S0022-460X(02)00920-3.

2. Fernandez del Rincon, A.; Viadero, F.; Iglesias, M.; García, P.; de-Juan, A.; Sancibrian, R. 2013. A model for the study of meshing stiffness in spur gear transmissions, Mechanism and Machine Theory 61: 3058. 
http://dx.doi.org/10.1016/j.mechmachtheory.2012.10 .008 .

3. Eritenel, T.; Parker, R.G. 2012. An investigation of tooth mesh nonlinearity and partial contact loss in gear pairs using a lumped-parameter model, Mechanism and Machine Theory 56: 28-51. http://dx.doi.org/10.1016/j.mechmachtheory.2012.05 .002 .

4. Zhang, Y.; Wang, Q.; Ma, H.; Zhao, C. 2013 Dynamic analysis of three-dimensional helical geared rotor system with geometric eccentricity, Journal of Mechanical Science and Technology 27(11): 32313242. http://dx.doi.org/10.1007/s12206-013-0846-8.

5. Palermo, A.; Mundo, D.; Hadjit, R.; Desmet, W. 2013. Multibody element for spur and helical gear meshing based on detailed three-dimensional contact calculations, Mechanism and Machine Theory 62: 1330 .

http://dx.doi.org/10.1016/j.mechmachtheory.2012.11 .006 .

6. Huang, K.J.; Su, H.W. 2010. Approaches to parametric element constructions and dynamic analyses of spur/helical gears including modifications and undercutting, Finite Elements in Analysis and Design 46(12): 1106-1113. http://dx.doi.org/10.1016/j.finel.2010.08.002.

7. Fuentes, A.; Nagamoto, H.; Litvin, F.L.; GonzalezPerez, I.; Hayasaka, K. 2010. Computerized design of modified helical gears finished by plunge shaving, Computer Methods in Applied Mechanics and Engineering 199(25-28): 1677-1690. http://dx.doi.org/10.1016/j.cma.2010.01.023.

8. Nishino T. 2007. Integrated excitation models of the helical gear system, 10th International Power Transmission and Gearing Conference 7: 477-486.

9. Sondkar, P.; Kahraman, A. 2013. A dynamic model of a double-helical planetary gear set. Mechanism and Machine Theory 70: 157-174. http://dx.doi.org/10.1016/j.mechmachtheory.2013.07 .005 .

10. Cooley, C.G.; Parker, R.G.; Vijayakar, S.M. 2010. An efficient finite element solution for gear dynamics, IOP Conference Series: Materials Science and Engineering 10(1): 1-10. http://dx.doi.org/10.1088/1757-899x/10/1/012150.

11. Velex, P.; Bruyere, J.; Houser, D.R. 2011. Some Analytical Results on Transmission Errors in Narrow-
Faced Spur and Helical Gears: Influence of Profile Modifications, Journal of Mechanical Design 133(3): 111. http://dx.doi.org/10.1115/1.4003578.

12. Nasisi S.; Valasek M.; Vampola T. 2015. An Interpolation Method for Determining the Frequencies of Parameterized Large-Scale Structures, Acta Polytechnica 55(6): 407-414.

https://doi.org/10.14311/AP.2015.55.0407.

13. Neusser, Z.; Sopouch, M.; Schaffner, T.; Priebsch, H.-H. 2010. Multi-body Dynamics Based Gear Mesh Models for Prediction of Gear Dynamics and Transmission Error, in Transmission and Driveline, Detroit, SAE International. http://dx.doi.org/10.4271/2010-01-0897.

\section{Z. Neusser, T. Vampola, M. Valášek}

\section{ANALYTICAL GEAR MESH MODEL USING 3D GEAR GEOMETRY}

S u m m a r y

This paper presents a model of the interaction of mating gears. The approach uses 3D gear geometry for the calculation of teeth penetration. The contact force is evaluated using the penetration between all mating teeth and the known contact stiffness. Gear meshing is evaluated using an analytical solution based on gear flank parameterization. The advantage of such an approach is to involve real gear geometry in the calculation of the contact force. The model involves the effects of the backlash between teeth (gear rattling, hammering), engagement and disengagement of the teeth pair in contact and the variation of the number of teeth pairs in contact (gear whine). The ideal tooth flank geometry is modified using the measured flank modifications. The dynamic behaviour of the interaction between two gears is modelled and compared with the 2D spring-damper model.

Keywords: gear mesh model, 3D gear geometry, gear contact forces, tooth flank modification.

Received March 22, 2016

Accepted June 08, 2017 